\title{
Automation of the evaluation process of technical condition and repair planning of freight wagons using computer tools
}

\author{
Marek Płaczek* \\ Silesian University of Technology, Faculty of Mechanical Engineering, Institute of Engineering \\ Processes Automation and Integrated Manufacturing Systems, Konarskiego 18A, 44-100 Gliwice, \\ Poland
}

\begin{abstract}
Freight wagons are verified about their technical condition at a certain time interval and during this qualification process a standard paper qualifying protocol is used. It is necessary to carry out a more accurate operational documentation in order to obtain more precise information about the process of damage of individual elements. This documentation should be created starting from the production of wagons. The automation of this process using computer tools is presented. A ready to use solution is proposed.
\end{abstract}

\section{Introduction and aim of the research works}

Development of all technical devices and technology using computer aided method is the main idea of research works conducted by scientist all over the world [1-5].

This paper contains a report on the part of works conducted in the research and development project entitled "Analytical and experimental studies and determination of the structural features of components and assemblies in innovative structure of repaired wagons". This project was realized within the Program of Applied Research by Institute of Engineering Processes Automation and Integrated Manufacturing Systems of Silesian University of Technology together with consortium partners: company DB Schenker Rail Poland SA and Germaz. The main objective of the project was to develop a technology of modernization of freight wagons for the transport of coal and aggregates, through the use of innovative materials and technologies to repair this type of wagons during periodic repairs. The main aim of the project was to improve the operating conditions of considered types of freight wagons. To increase their resistance to corrosion and freezes transported cargo to the shell of the body in the winter conditions, and thus an easier unloading as well as verification of strength of modernized carriages and an estimation of the possibility of reducing their weight, while maintaining or increasing the permissible load [6-8].

One of elements of the project was also to develop a system for diagnosing the technical condition of the modernized shell of wagon body during operation. For this purpose the use of non-destructive testing methods of technical state of constructions was used, including

* Corresponding author: marek.placzek@polsl.pl 
methods that use the analysis of dynamic response of the object. Therefore research which examines the possibility of use of the foils as sensors in the system of vibration measurement of tested items were conducted [9]. Application of the composite panels to the freight wagon's body shell was proposed as the solution that can solve mentioned problems during exploitation of freight wagons [6-10]. The composite panels composed of fiberglass and epoxy resin were proposed. They were mounted on the body shell using rivet nuts. What is more the body shell of the modernized freight wagon was painted using an anti-corrosion agent, while the standard freight wagon body is not painted inside at all.

In the research project a statistical analysis of data from renovations of freight wagons was done. At this moment the qualifying protocol that is used in the DB Schenker Rail Poland SA Company in order to decide which elements of the freight wagon should be repaired or replaced by the new ones includes such data as:

- $\quad$ the type of the analysed freight wagon;

- drawings of the analysed type of wagons including views of all walls and the floor;

- $\quad$ the identification number of the wagon;

- $\quad$ the date and type of the previous repair and the date and type of the actual renovation;

- the table with listed elements of the wagon with the possibility to mark whether the item is suitable for repair, replacement, or whether it is in good technical condition;

- $\quad$ signatures of responsible persons and the place for other notes.

A statistical analysis of data from qualifying protocols were analysed in order to verify if some wagon's elements are the most exposed to damage during exploitation of freight wagons [10]. Qualifying protocols from years 2012 up to 2014 were analysed. The percentage of repairs of individual wagons elements was specified on the basis of protocols analysis.

The most important, taking into account the aim of the research work - the modernization of the freight wagons using composite panels mounted to their body shell, was the information about the damage of its elements but results of qualifying protocols analysis showed that there is no any part of the freight wagons body shell that may be indicated as the most exposed to damage during standard exploitation of the freight wagons.

It should be mentioned that in order to obtain more precise information about the process of damage of individual elements of the freight wagons body shell during their exploitation it is necessary to carry out a more accurate operational documentation starting from the production of wagons. This documentation should be created starting from the production of wagons and it should take into account operating conditions of carriage, such as the type of transported cargo, as well as all carried out repairs.

\section{Automation of the evaluation process of freight wagons}

The possibility of development of the process of qualifying freight wagons to repairs as well as the whole process of their repairing using computer aided systems was also an aim of this research project. The aim of the study was the use of selected virtual environments to support the process of repair of freight wagons and analysis of project-based approach to this issue, based on qualifying protocols shared by DB Schenker Rail Poland SA.

Assessment of the technical condition of a freight wagon is complex and time-consuming. It consists of several stages. The qualifying protocol must be filled in with information about the date and place of qualifying process, the type and number of the freight wagon. Then, data about freight wagon's elements are entered - data about wheelset, trolley, sheathing of the wagon's body and others. It is important that measured values of thickness of all elements must be written down. The number of the sheathing elements is about twenty-five depending on the type of the freight wagon that is being serviced. The thickness of each element is measured in two places - on the top and bottom of the plate of the sheathing. Each element 
should be qualified if it is in good condition or should be repaired or replaced by the new one. This is why the qualifying process can be considered as uncomfortable manual paper work and very often the documentation was filled carelessly. If someone wanted to introduce changes he had to write down the changes on paper, which unfortunately is often associated with the fact that the document was unreadable.

In order to improve the work of technicians during the qualifying process of freight wagons, to be able to quickly and easily enter data and to archive the data in one file (making possible a detailed analysis of repairs) two applications were created.

The mobile application for Android system in order to enter and send data is the first. The second one - a web application used for receiving data from the tablet. By using it, data can be presented in the form of a spreadsheet with the option to download in CSV format. Created protocol can be opened in programs such as Microsoft Excel, Open Office Calc or text editor. The aim of the study was to write applications for the Android device in conjunction with the possibility to write data to a spreadsheet. The application was written to create a separate file, accessible to all users, which will be the result of recording the data entered into the application. In order to use the application and record the results the device need to be connected to the Internet.

Due to the rapid development of new technologies and mobile devices such as tablets and smartphones, the application was written for the tablet with the most popular mobile operating system that is Android. The program works well with the version of the API level 15 - a version number 4.0.3. Below this version there is no guarantee that the application will run correctly. To write a mobile application the Java language was used with the tool developed to build such projects called Gradle. Therefore, the device do not need to store any data (collected only during fulfilling the sheets and forwarded) is not used there natively embedded in the Android SQLite data base [11-13].

When creating the application to store data between screens a mechanism called Shared Preferences was used. This mechanism allows keeping a small amount of simple data types such as integer or string. In the case of the collection of data fields a class object StringBuilder was used. For providing Internet connection and send data using POST method the class HttpURLConnection was used.

To create a web application the PHP programming language, the MySQL database, the library jQuery and the CSS Twitter bootstrap framework were used. The PHP programming language is one of the most popular languages for building web pages and frequently to cooperate with it the MySQL database is used [11-13].

The first step after starting the application is login of the user. To add a new user one must enter it to the list in the application code. This prevents the application from creating additional accounts by unauthorized persons. Additionally, on each screen and each card of the application in places where one can enter the text a standard keyboard "qwerty" is available. After a successful login to your account application goes to the main menu, which appears on the left side of the display. At this point the user of the application has a choice of sections such as data, wheelset, trolley, sheathing of the wagon body and others. Each section runs as a "checklist", so there is no possibility that while switching windows any data will be lost.

After selecting the appropriate section the user is able to supplement the data according to the physical condition of a classified freight wagon. It is necessary to introduce the type of wagon, wagon number, type/series, the last type of repair etc. also the user has the opportunity of note comments.

After completing all sections and addition of comments the user clicks on the icon in the lower right corner and the application automatically sends and saves generated qualifying protocol. 


\section{The project approach to the freight wagons renovation process}

Technological processes and management in large enterprises are based on the project approach to problems. To achieve a higher degree of management process tools supported on computer systems are most commonly used [14-16].

Market demand for such services has resulted in a large amount of software in a broader or less degree supporting the processes of production management, web services, repair maintenance services and as a result, maintenance of external resources, internal resources and control of process lines. Depending on the size of the enterprise and approach to the management more or less developed and powerful tool can be applied. Its goal is to ensure increased productivity, control and reduce operating costs.

The most common systems supporting production processes and technology are systems CMMS (Computerized Maintenance Management Systems). These tools work with other systems via bus integration tools such as ERP (SAP) and surveillance systems of technological processes (SCADA).

In the research project the use of IBM Maximo Asset Management to manage the qualification process and repair of freight wagons was proposed $[15,16]$. It was assumed design approach to managing the process of repairing a freight wagons. The result of the performed simulation was the rise of a project in which all elements relating to the process flow of information related to the classification of repairing a freight wagon were introduced. In this process of the project approach to the issue of supervision a collective summary of information on the implementation of the objective pursued and the costs incurred at him were received.

From the project it is possible to perform multiple operational reports on project tasks, work orders, distributed cost, ordered materials and services, and the status of their advancement.

In Figure 1 assumption of the information flow of work orders in proposed project is presented. A scheme of the process of repair or maintenance of a freight wagon is presented in Figure 2.

In addition, the possibility of developing the system for classification of freight wagons to repair in a system SIMPLE.ERP was analysed. Key benefits that entail the use of the proposed classification system are:

- The ability to use the system SIMPLE.ERP on a mobile device such as tablet. The user can enter data on a regular basis during the inspection of the wagon. The entered information, at the same time can be seen in other places in the enterprise, for example in the planning department or in the warehouse. This gives the ability to quickly respond to the demand.

- Classification system created in SIMPLE.ERP deprives employees need to use multiple sheets of repairs card. It causes savings and no need to archive multiple documents. It also facilitates access to the history of performed operations that are archived in the form of data on the server.

- It gives the ability to quickly analyse data by exporting data to a Microsoft Excel file, what in the case of traditional repairs cards was impossible.

- With a single program, the operator is able to classify the data, at the same time, check availability of the part in the module "magazine" and in the case of their absence, order them using another module. All these activities, the operator can perform using a single application. 


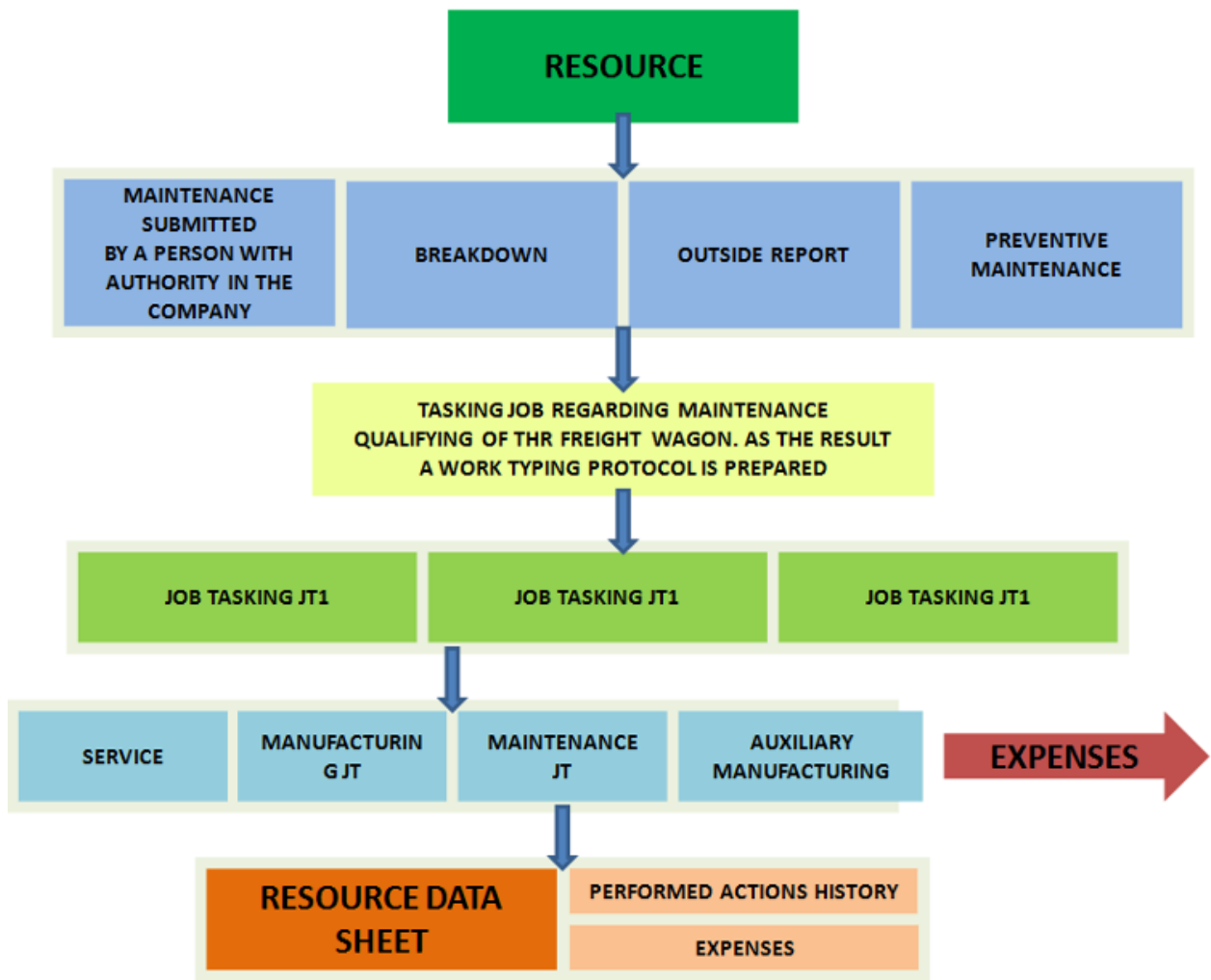

Fig. 1. The information flow of work orders.

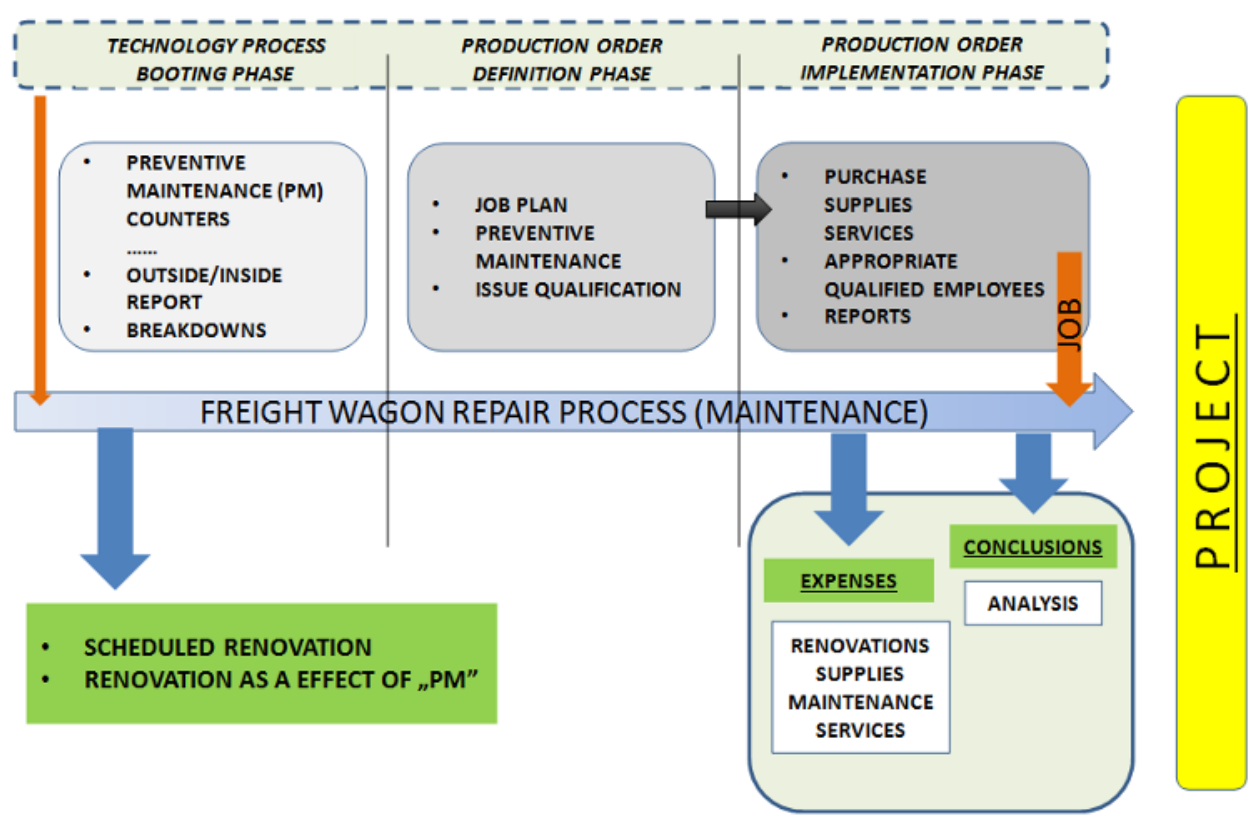

Fig. 2. A scheme of the process of repair or maintenance of a freight wagon. 


\section{Conclusions}

As a response to the needs of the industry company the application to support the process of collecting and analysing data needed for planning of the freight wagons renovation process was created. With this application the process, which until now was entirely physically performed by employees can be automated. Entering data is divided into several stages, between which you can move flexibly and freely edit them - the blurring of the old data with new ones was removed - everything is clear. After all the fields were filled, just press a button, and all the data will be sent to the server.

The second application is installed on the server, which receives the data and presents it in the similar form as before - on the standard qualifying protocols, making it visually does not differ from what is known to employees. At the same time the computer data storage system was introduced - so far there was the need to store a lot of paper documents. Another advantage is no need to involve an additional employee to the time-consuming operations of rewriting data sheets (often illegible). With the introduction of this automated process analysis and qualifying of the wagons there is also a possibility of easily examination and comparing of the incoming data. It will allow for an even better future work and planning of repairs and the purchase of parts.

The project approach to the freight wagons renovation process was also proposed. An application of computer aided systems for supporting the processes of production management, web services, repair maintenance services and, as a result, maintenance of external resources, internal resources and control of process lines were considered. The proposed solutions can bring a lot of benefits to the process of freight wagons qualifying and their renovation process.

\section{References}

1. K. Jamroziak, M. Bocian, M. Kulisiewicz, Mechanics, 19(5), 549-553 (2013)

2. P. Ociepka, K. Herbuś, A. Gwiazda, Adv. Materials Research 1036,1011-1016 (2014)

3. E. Rusiński, S. Dragan, P. Moczko., Pietrusiak D., Arch. of Civil and Mechanical Eng., 12, 471-476 (2012)

4. A. Buchacz, A. Wróbel, Solid State Phenomena, 164, 239-242 (2010)

5. A. Wróbel, J. of Vibroengineering, 14 (2), 534-537 (2012)

6. A. Wróbel, M. Płaczek, A. Buchacz, M. Majzner, Int. J. Materials and Product Technology, 50 (3/4), 259-275 (2015)

7. M. Płaczek, A. Wróbel, A. Baier, IOP Conf. Ser.: Mater. Sci. Eng. 145, 042026 (2016)

8. M. Płaczek, A. Wróbel, A. Buchacz, IOP Conf. Ser.: Mater. Sci. Eng. 145, 072012 (2016)

9. M. Płaczek, A. Buchacz, A. Wróbel, Eksploatacja i Niezawodnosc - Maintenance and Reliability 17, 443-449 (2015)

10. M. Płaczek, A. Wróbel, A. Buchacz, IOP Conf. Series: Materials Science and Engineering 161, 012107 (2016)

11. E. Hellman, Android platform. New challenges (Helion, Warsaw, 2014, In Polish)

12. J. Annuzzi jr., L. Darcey, S. Conder, Introduction to Application Programming (Helion, Warsaw, 2016), In Polish

13. R. Nixon, PHP, MySQL and JavaScript. Introduction (Helion, Warsaw, 2015)

14. K. Kalinowski, C. Grabowik, W. Kempa, I. Paprocka, Advanced Materials Research, 837, 422-427 (2014)

15. S. Krajewski, Z. Banaszak, Wiadomosci Gornicze, 61 (2), 107-113 (2010), In Polish

16. W. Puchała, W. Biały, G. Bobkowski, Scientific Papers of Maritime Academy in Szczecin, 5(77), 403-412 (2015), In Polish 